\title{
Effect of Immediate Dentin Sealing and Surface Conditioning on the Microtensile Bond Strength of Resin-Based Composite to Dentin
}

\author{
CRG van den Breemer • M Özcan • MS Cune \\ AP Almeida Ayres • B Van Meerbeek • MMM Gresnigt
}

\begin{abstract}
Clinical Relevance
For partial indirect restorations, immediate dentin sealing is recommended, as bond strength remains stable over time.
\end{abstract}

\section{SUMMARY}

This study evaluated the microtensile bond strength ( $\mu$ TBS) of resin-based composite (RBC) to dentin after different immediate dentin sealing (IDS) strategies and surfaceconditioning (SC) methods and on two water storage times. Human molars $(n=48)$ were randomly divided into eight experimental

*Carline van den Breemer, DDS, research fellow, University Medical Center Groningen, Center for Dentistry and Oral Hygiene, Department of Fixed and Removable Prosthodontics and Biomaterials, University of Groningen, Groningen, Netherlands

Mutlu Özcan, DDS, PhD, professor, Division of Dental Materials, Center for Dental and Oral Medicine, Clinic for Fixed and Removable Prosthodontics and Dental Materials Science, University of Zurich, Zurich, Switzerland

Marco Cune, DDS, PhD, professor, St Antonius Hospital Nieuwegein, Department of Oral-Maxillofacial Surgery, Prosthodontics and Special Dental Care, Nieuwegein, Netherlands, and University Medical Center Utrecht, Department of Oral Maxillofacial Surgery, Prosthodontics and Special Dental Care, Utrecht, Netherlands

Ana Paula Almeida Ayres, DDS, PHD, Department of Restorative Dentistry, University of Uberaba, Uberaba, Brazil groups involving four different IDS strategies-IDS-1L with one layer of adhesive, IDS2L with two layers of adhesive, IDS-F with one layer of adhesive and one layer of flowable RBC, and DDS (delayed dentin sealing) with no layer of adhesive (control)_and two different SC methods-SC-P with pumice rubbing and SC-PC with pumice rubbing followed by tribochemical silica coating. The $\mu$ TBS test was

Bart van Meerbeek, DDS, PhD, professor, Department of Oral Health Sciences, BIOMAT, KU Leuven (University of Leuven), and University Hospitals Leuven, Dentistry, Leuven, Belgium

Marco Gresnigt, DDS, PhD, Martini Hospital, Department of Prosthodontics and Special Care, Groningen, Netherlands, and University Medical Center Groningen, Center for Dentistry and Oral Hygiene, Department of Fixed and Removable, Prosthodontics and Biomaterials, Groningen, Netherlands

*Corresponding author: University Medical Center Groningen, Center for Dentistry and Oral Hygiene, Department of Fixed and Removable Prosthodontics, Antonius Deusinglaan 1, 9713 AV, Groningen, Netherlands; e-mail: c.r.g. van.den.breemer@umcg.nl

DOI: http://doi.org/10.2341/18-052-L 
performed after one week and after six months of water storage, being recorded as the "immediate" and "aged" $\mu$ TBS, respectively. Composite-adhesive-dentin microspecimens $(0.9 \times 0.9 \times 8-9 \mathrm{~mm})$ were stressed in tension until failure to determine the $\mu$ TBS. Failure mode and location of failure were categorized. Twoway analysis of variance was applied to analyze the data for statistically significant differences between the experimental groups $(p<0.05)$. Two-way analysis of variance revealed no significant differences between the one-week $\mu$ TBS specimens for IDS strategy $(p=0.087)$ and SC methods $(p=0.806)$. However, the interaction of IDS strategy and SC methods appeared statistically significant $(p=0.016)$. The six-month specimen evaluation showed no significant difference in $\mu$ TBS for SC $(p=0.297)$ and SC/IDS interaction $(p=0.055)$, but the $\mu$ TBS of the IDS strategies differed significantly among them $(p=0.003)$. For tribochemical silica-coated IDS, no significant effect of aging on $\mu$ TBS was recorded $(p=0.465)$, but there was a highly significant difference in $\mu$ TBS depending on the IDS strategy $(p<0.001)$. In addition, the interaction of IDS and aging was borderline statistically significant $(p=0.045)$. The specimens failed mainly at the adhesive-dentin interface for all experimental groups. Dentin exposure during clinical procedures for indirect restorations benefits from the application of IDS, which was shown to result in higher bond strength. No significant differences were found between cleaning with solely pumice or pumice followed by tribochemical silica coating.

\section{INTRODUCTION}

In restorative dentistry, one of the primary goals is to preserve tooth tissue. Removing large amounts of dental structure adversely affects the pulp and may lead to pulp damage. ${ }^{6,20}$ When it comes to restoring posterior teeth with large cavities, partial indirect restorations in glass-ceramic or feldspathic porcelain may be indicated. The literature reveals that such restorations have a survival rate of $91 \%$ in 10 years. ${ }^{24}$ The cause of failure involves fractures (4\%), endodontic complications (3\%), secondary caries $(1 \%)$, and debonding $(1 \%) .{ }^{24}$ Fractures and debonding are often seen in cases where restorations are bonded to dentin. ${ }^{13}$ Creating a strong bond to dentin that is durable over time is more challenging than creating a bond to enamel because of dentin's intrinsic hydrophilic nature. ${ }^{3}$ An inadequate seal of dentin by the adhesive may cause postoperative sensitivity, marginal staining, and recurrent caries. Hence, the survival and success of (partial) indirect restorations is often related to the remaining quantity and quality of enamel. ${ }^{15}$

In order to improve bond strength to dentin, Pashley and others introduced the so-called dual bonding technique, which consists of the application of two layers of adhesive resin onto dentin. ${ }^{26}$ Applying an adhesive layer directly after crown preparation protects the pulp from bacterial invasion, reduces postoperative sensitivity, and increases bond strength. Other studies revealed that multiple adhesive layers can further improve bond quality and strength. ${ }^{8,17,19,27}$ The purpose of sealing dentin directly after preparation is to avoid surface contamination during the temporary phase and to protect dentin by hybridization, thus avoiding sensitivity and preventing water uptake. This requires that the adhesive be light cured immediately, which is commonly not recommended at the time of cementation to avoid restoration fit problems. ${ }^{32}$

In 2005, this concept evolved to immediate dentin sealing (IDS). Prior to luting in the second visit, one commonly recommends decontaminating the IDS by tribochemical silica coating. ${ }^{21,22}$ This not only microroughens the surface, thereby improving micromechanical interlocking, but also cleans the surface and enables chemical copolymerization of the resinbased cement with the IDS. ${ }^{1,33,37}$ Falkensammer and others $^{14}$ concluded that polishing and airborne particle abrasion with silica-coated alumina $\left(\mathrm{Al}_{2} \mathrm{O}_{3}\right)$ and glycine are equally efficient methods of conditioning IDS surfaces. Other studies showed that soft air abrasion, ${ }^{34}$ airborne particle abrasion with $\mathrm{Al}_{2} \mathrm{O}_{3},{ }^{11,22,23}$ or fluoride-free pumice paste systems ${ }^{5,12,21}$ resulted in the highest bond strength. However, it is unknown which method is most suitable for conditioning IDS prior to cementation.

Results from a recent systematic review indicated that the effect of IDS on bond strength is tested mainly by using a microtensile bond strength ( $\mu$ TBS) approach. ${ }^{38}$ The $\mu$ TBS test is generally accepted as one of the most valid bond-strength tests because it is performed perpendicular to the adhesive interface. ${ }^{10,35}$ Using a $\mu$ TBS test, a more favorable stress distribution is achieved, resulting from the small specimen size.

The objective of this study was to compare different IDS applications and surface conditioning 

Table 1: $\begin{aligned} & \text { Brand, Manufacturer, Main Chemical Composition, and Batch Number of the Different Products Used (in Alphabetical } \\ & \text { Order) }\end{aligned}$

\begin{tabular}{|c|c|c|}
\hline $\begin{array}{c}\text { Product } \\
\text { (Manufacturer) }\end{array}$ & Composition & $\begin{array}{l}\text { Batch } \\
\text { Number }\end{array}$ \\
\hline $\begin{array}{l}\text { CoJet sand ( } 3 \mathrm{M}, \text { Seefeld, } \\
\text { Germany) }\end{array}$ & Aluminum oxide $\left(\mathrm{Al}_{2} \mathrm{O}_{3}\right)$ particles coated with silica (particle size: $30 \mu \mathrm{m}$ ) & $446317 / 534151$ \\
\hline \multirow[t]{2}{*}{ Durelon (3M) } & Powder: zinc oxide, stannous fluoride, tin dioxide & 514362 \\
\hline & Liquid: water, polyacrylic acid & 510198 \\
\hline $\begin{array}{l}\text { Enamel plus HFO UD2 } \\
\text { (Micerium, Avegno, Italy) }\end{array}$ & 1,4-Butandioldimethacrylate, urethane dimethacrylate, Bis-GMA & 2015007203 \\
\hline ESPE-Sil (3M) & Ethyl alcohol, 3-methacryloxypropyl- trimethoxysilane, methyl ethyl ketone & 598868 \\
\hline $\begin{array}{l}\text { Glycerin Gel K-Y, } \\
\text { (Johnson \& Johnson, } \\
\text { Sezanne, France) }\end{array}$ & $\begin{array}{l}\text { Purified water, glycerin, methylparaben, propylparaben, propylene glycol, } \\
\text { hydroxyethylcellulose, dissodium phosphate, sodium phosphate, tetrasodium EDTA }\end{array}$ & $2744 \mathrm{~V}$ \\
\hline $\begin{array}{l}\text { Grand IO Flow (VOCO, } \\
\text { Cuxhaven, Germany) }\end{array}$ & 1,6-Hexanediylbismethacrylate, BIS GMA, triethylene glycol dimethacrylate & 1512472 \\
\hline $\begin{array}{l}\text { Monobond Plus (Ivoclar } \\
\text { Vivadent, Schaan, } \\
\text { Liechtenstein) }\end{array}$ & Ethanol, 3-trimethoxysilsylpropyl-methacrylate, methacrylated phosphoric acid ester & T07775 \\
\hline \multirow{2}{*}{$\begin{array}{l}\text { Optibond FL (Kerr, } \\
\text { Orange, CA, USA) }\end{array}$} & Primer: HEMA, GPDM, PAMM, ethanol, water, photoinitiator & 5534310 \\
\hline & Adhesive: TEGDMA, UDMA, GPDM, HEMA, bis-GMA, filler, photoinitiator & 5594053 \\
\hline $\begin{array}{l}\text { Total Etch (Ivoclar } \\
\text { Vivadent) }\end{array}$ & $37 \%$ phosphoric acid $\left(\mathrm{H}_{3} \mathrm{PO}_{4}\right)$ & L049 \\
\hline
\end{tabular}

(SC) methods by determining the bond strength to dentin with and without water storage aging. The null hypotheses tested were that (1) there is no significant difference in $\mu$ TBS among the four IDS strategies investigated, (2) among the two SC methods, and (3) for the "immediate" (one-week) and "aged" (six-month) $\mu$ TBS.

\section{METHODS AND MATERIALS}

\section{Specimen Preparation}

Human molars $(n=48)$ were collected, stored in distilled water, and used at maximum one month postextraction. Each specimen was embedded in gypsum to facilitate handling. The occlusal coronal third of the crown was removed with a diamond saw (Isomet 1000, Buehler, Lake Bluff, IL, USA), thereby exposing a flat midcoronal dentin surface. The dentin surfaces were verified for the absence of enamel and/or pulp tissue exposure using a stereomicroscope (Wild M5A, Wild, Heerbrugg, Switzerland).

\section{Study Design}

The flattened specimens were randomly divided into a total of eight experimental groups involving four different IDS strategies-IDS-1L with one layer of adhesive, IDS-2L with two layers of adhesive, IDS-F with one layer of adhesive and one layer of flowable resin-based composite (RBC), and DDS (delayed dentin sealing) with no layer of adhesive (control) and two different SC methods-SC-P with pumice rubbing and SC-PC with pumice rubbing followed by tribochemical silica coating. The $\mu$ TBS test was performed after one week and after six months of water storage, being recorded as the "immediate" and "aged" $\mu \mathrm{TBS}$, respectively.

\section{IDS}

The brand, manufacturer, main chemical composition, and batch number are detailed in Table 1 for the different products used in this study.

Regarding IDS-1L, the flat dentin was etched for 15 seconds with 37\% phosphoric acid (Total Etch, Ivoclar Vivadent, Schaan, Liechtenstein) and rinsed thoroughly with a water-air spray for 15 seconds. The surface was air-dried but not desiccated for 3 seconds, after which a primer (Optibond FL Primer, Kerr, Orange, CA, USA) was applied with a light brushing motion for 15 seconds and gently air-dried for 10 seconds and suction dried for 15 seconds. A thin layer of heated $\left(40^{\circ} \mathrm{C}\right)$ adhesive resin (Optibond FL Adhesive, Kerr) was applied onto the surface using a light brushing motion for 15 seconds and 
photopolymerized for 10 seconds (Bluephase Style, Ivoclar Vivadent) $\left(>1000 \mathrm{~mW} / \mathrm{cm}^{2}\right)$. Regarding IDS$2 \mathrm{~L}$, the same procedures were performed with the addition that a second layer of adhesive was likewise applied as described above for the first adhesive layer. Regarding IDS-F, the same procedure was performed as for IDS-1L with the addition that a flowable RBC (Grand IO Flow, VOCO, Cuxhaven, Germany) was additionally applied in a thin layer of about an average thickness of $1 \mathrm{~mm}$ and separately light cured for 40 seconds. Finally, in all groups, glycerin gel (K-Y, Johnson \& Johnson, Sezanne, France) was applied, and light curing was repeated for 40 seconds.

The DDS was considered the control; the dentin was not sealed directly after preparation.

\section{Temporary Restoration}

After dentin preparation, a temporary restoration (Protemp 4, 3M, Seefeld, Germany) was cemented onto the flat dentin surface using a zinc-carboxylate cement (Durelon, 3M). The temporary phase consisted of three weeks of water storage.

\section{Surface Conditioning and Composite Buildup}

After three weeks, the temporary restorations were removed with a scaler (H5 Anterior Scaler, HuFriedy, Chicago, IL, USA). Next, the surfaces of half of the specimens for each of the four IDS groups were cleaned solely by pumice rubbing (SC-P), while those of the other half of the specimens were cleaned by pumice rubbing and additionally tribochemically silica coated (SC-PS) at a distance of 10 $\mathrm{mm}$ following a $45^{\circ}$ angulation ( 2 bar; CoJet sand, $\left.\mathrm{SiO}_{2}, 3 \mathrm{M}\right)$. All specimens were subsequently rinsed thoroughly with water and air-dried for 15 seconds, after which a silane coupling agent (ESPE-SIL, 3M) was applied and left to dry (five minutes) according to the method of Ozcan and others. ${ }^{25}$ The primer (Optibond FL Primer, Kerr) was then applied onto all specimen surfaces with a light brushing motion for 15 seconds, gently air-dried for 10 seconds, and then suction dried for 15 seconds. A thin layer of heated $\left(40^{\circ} \mathrm{C}\right)$ adhesive resin (Optibond FL Adhesive, Kerr) was next applied onto the surface with a light brushing motion for 15 seconds and air-dried for 10 seconds without light curing. An RBC buildup $( \pm 6 \mathrm{~mm})$ was subsequently constructed (HFO composite, Micerium, Avegno, Italy) on the prepared surfaces by filling a transparent silicon mold in three to four layers. Caution was taken to avoid air bubbles between the adhesive and RBC layers. The buildup was photopolymerized through the silicon mold using the LED light-curing unit from above and after the last layer it was lightcured for 40 seconds from each of the four sides and from the top; this was repeated after having removed the mold and having applied Glycerin Gel (Johnson \& Johnson) at the RBC surface outside.

\section{Aging and $\mu$ TBS Testing}

After one week of storage in $0.5 \%$ chloramine $\mathrm{T}$ solution at $37^{\circ} \mathrm{C}$, microspecimens were cut for $\mu$ TBS testing. Per experimental group, 24 microspecimens were tested immediately to measure the one-week $\mu$ TBS, while another set of 24 microspecimens were tested after six months of storage to measure the sixmonth aged $\mu$ TBS. The bond strength to dentin was determined using a standardized $\mu$ TBS protocol. ${ }^{31} \mathrm{In}$ order to obtain 12 rectangular microspecimen sticks per experimental group $(0.9 \times 0.9 \times 8-9 \mathrm{~mm})$, the restored teeth were sectioned perpendicular to the interface using an automated water-cooled precision diamond saw (Accutom-50, Struers, Ballerup, Denmark). ${ }^{30,40}$ The dimensions of the sticks were precisely measured by means of a digital caliper (CD-15CPX, Mitutoyo, Kanagawa, Japan) from which the cross-sectional area was calculated $(\sim 0.9$ $\mathrm{mm}^{2}$ ). The microspecimens were fixed to a modified $\mu$ TBS testing jig $^{31}$ using cyanocrylate glue (Model Repair II Blue, Dentsply-Sankin, Tokyo, Japan) and tested in tension mode at a crosshead speed of 1.0 $\mathrm{mm} / \mathrm{min}$ using an LRX testing machine (Lloyd, Hampshire, UK) equipped with a load cell of 100 $\mathrm{N}$. The bond strength values were calculated in $\mathrm{MPa}$ by dividing the imposed force (in $\mathrm{N}$ ) at the time of fracture by the bonded area (in $\mathrm{mm}^{2}$ ). Specimens that failed before actual testing (pretesting failure) were explicitly noted, counted as $0 \mathrm{MPa}$ in further analyses, and thus taken into account for the calculation of the $\mu$ TBS means.

\section{Failure Pattern Analysis}

The failure modes were evaluated using a stereomicroscope (Wild M5A, Wild) at a magnification of up to $50 \times$ and classified as follows: failure in dentin, failure at the adhesive-dentin interface, failure in the adhesive resin, failure at the adhesive-composite interface, and failure in the composite. Representative specimens in each group were selected for further ultrastructural characterization using scanning electron microscopy (SEM). The latter specimens were sputter coated using a 3-nm-thick layer of gold (80\%) and palladium (20\%) (90 seconds, $45 \mathrm{~mA}$; Balzers SCD 030, Balzers, Liechtenstein) prior to 
Table 2: $\quad$ Mean Microtensile Bond Strength (MPa) for the Different Immediate Dentin Sealing (IDS) and Surface-Conditioning (SC) Strategies at One Week and After Six Months of Aging ${ }^{a}$

\begin{tabular}{|c|c|c|c|c|}
\hline & IDS-1L & IDS-2L & IDS-F & DDS \\
\hline \multicolumn{5}{|l|}{$1 \mathrm{wk}$} \\
\hline SC-P & 29.8 (13.3) aA & 26.4 (11.2) aA & 29.1 (13.7) aA & 30.2 (17.1) aA \\
\hline SC-PS & $35.2(19.0) \mathrm{aA}$ & 39.2 (15.2) aB & 28.1 (13.6) aA & 16.0 (13.5) bB \\
\hline \multicolumn{5}{|l|}{$6 \mathrm{mo}$} \\
\hline SC-P & 29.5 (11.6) aA & 37.1 (13.9) aA & 27.9 (14.3) aA & 21.1 (17.0) bA \\
\hline SC-PS & 35.6 (7.3) aA & 29.3 (13.7) aA & 40.6 (14.9) aA & 21.4 (10.3) bA \\
\hline \multicolumn{5}{|c|}{$\begin{array}{l}{ }^{a} \text { Mean (standard deviation); same lowercase letters indicate absence of statistically significant difference in the rows, and same uppercase letters indicate absence of } \\
\text { statistically significant difference in the columns }(\mathrm{p}<.05) ; I D S-1 L \text {, IDS with one layer of adhesive; IDS-2L, IDS with two layers of adhesive; IDS-F, IDS with one layer of } \\
\text { adhesive and one layer of flowable RBC; DDS, delayed dentin sealing (control); SC-P, surface conditioning with pumice; SC-PS, SC followed by tribochemical silica } \\
\text { coating. }\end{array}$} \\
\hline
\end{tabular}

being examined using a cold field-emission SEM (LEO 440, Leo Electron Microscopy, Cambridge, $\mathrm{UK})$.

\section{Statistical Analysis}

All data were analyzed using a statistical software package (SPSS 22, PASW statistics 18.0.3, Quarry Bay, Hong Kong, China). Kolmogorov-Smirnov and Shapiro-Wilk tests were used to test for a normal distribution of the data. As the data were normally distributed, the data were divided in two experiments according to aging (one week, six months) and surface conditioning (SC-P, SC-PS). Two-way analysis of variance and post hoc testing were applied to verify possible differences among the groups for the parameters of IDS, SC, and aging on $\mu$ TBS. In all tests, $p<0.05$ was considered to be statistically significant.

\section{RESULTS}

The IDS strategy $(F[1,217.3]=2.265, p=0.087$, $\mathrm{n}^{2}=0.072$ ) and type of surface conditioning (SC) $\left(F[1,217.3]=0.061, p=0.806, \mathrm{n}^{2}=0.001\right)$ did not produce statistical difference after one week of water aging (Table 2). However, the interaction of IDS and
SC strategy was statistically significant $(F[3$, $\left.217.3]=3.649, p=0.016, \mathrm{n}^{2}=0.111\right)$. Hence, the magnitude of the difference between the two SC methods after one week of water aging depends on the IDS strategy that was applied. Silica-coated (SC-PS) specimens from IDS-2L showed the highest mean one-week $\mu$ TBS. The mean $\mu$ TBS was significantly higher (Figure 1) when silica coating (SC-PS) was used for this particular IDS strategy ( $F[1$, $217.3]=4.556, p=0.036, \mathrm{p}^{2}=0.049$ ). In contrast, following a DDS strategy, SC-PS achieved significantly lower $\mu$ TBS than SC-P $(F[1,217.3]=5.630, p=0.020$, $\left.\mathrm{n}^{2}=0.060\right)$.

After six months of water aging, SC did not significantly affect $\mu$ TBS $(F[1,173.6]=1.099$, $p=0.297, \mathrm{n}^{2}=0.012$ ), while the IDS strategy significantly influenced $\mu \mathrm{TBS}(F[3,173.6]=5.110, p=0.003$, $\mathrm{n}^{2}=0.148$ ). The interaction of IDS and SC was not significant $\left(F[3,173.6]=2.631, p=0.055, \mathrm{n}^{2}=0.082\right)$. Post hoc tests revealed that regardless of the type of SC, all IDS strategies differed significantly ( $p=0.001$ to $p=0.004$ ) from the DDS group but not from each other (Table 2; Figure 2).

Table 2 shows that the aging time ( $F[1$, $\left.200.3]=0.000, p=0.997, \mathrm{y}^{2}=0.000\right)$ and IDS strategy
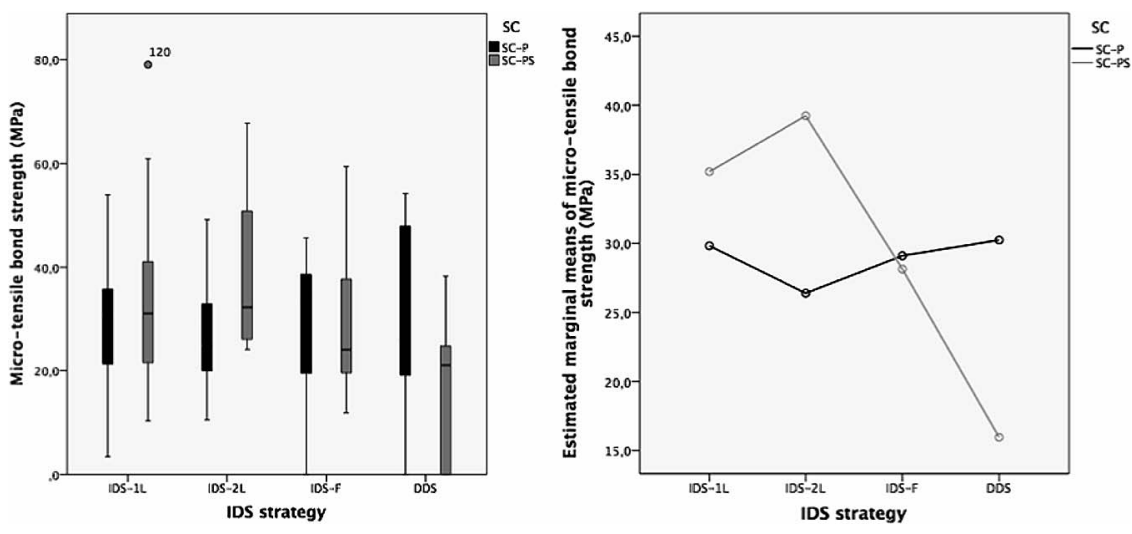

Figure 1. Box plot of microtensile bond strength (MPa) (left) and estimated marginal means of microtensile bond strength (MPa) (right) for the different immediate dentin sealing (IDS) and surface-conditioning strategies at one week. IDS-1L, IDS with one layer of adhesive; IDS-2L, IDS with two layers of adhesive; IDS- $F$, IDS with one layer of adhesive and one layer of flowable RBC; DDS, delayed dentin sealing (control); $S C$ $P$, surface conditioning with pumice; SC-PS, SC followed by tribochemical silica coating. 

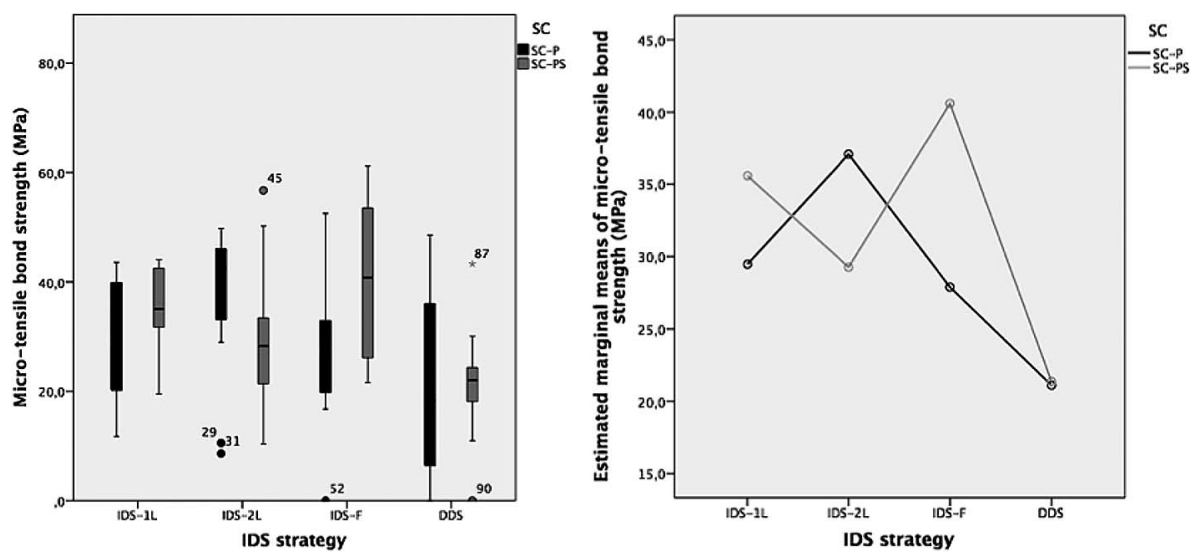

Figure 2. Box plot of microtensile bond strength $(\mathrm{MPa})$ (left) and estimated marginal means of microtensile bond strength (MPa) (right) for the different immediate dentin sealing (IDS) and surface-conditioning strategies after six months of aging. IDS-1L, IDS with one layer of adhesive; IDS$2 L$, IDS with two layers of adhesive; IDS-F, IDS with one layer of adhesive and one layer of flowable RBC; $D D S$, delayed dentin sealing (control); SC$P$, surface conditioning with pumice; SC-PS, SC followed by tribochemical silica coating.

$\left(F[3,200.3]=0.765, p=0.517, \mathrm{p}^{2}=0.025\right)$ did not produce statistical difference for the SC-P groups. There was no interaction of these factors as well $\left(F[3,200.3]=1.989, p=0.121, \mathrm{n}^{2}=0.064\right)$. However, in general, the SC-P groups demonstrated stable $\mu$ TBS means in time without significant differences among groups (Figure 3 ).

For the SC-PS specimens, the aging factor produced no significant difference $(F[1,190.6]=0.538$, $p=0.465, \mathrm{j}^{2}=0.006$ ). However, there was a highly significant difference in mean $\mu$ TBS among the IDS strategies $\left(F[3,190.6]=8.097, p<0.001, \mathrm{n}^{2}=0.216\right)$. DDS surfaces conditioned with silica (SC-PS) exhibited the significantly lowest $\mu$ TBS $(p<0.001$ for all pairwise comparisons). The aging/IDS strategy interaction was also statistically significant $(F[3$, 190.6] $=2.801, p=0.045, \mathrm{\eta}^{2}=0.087$ ). Hence, for SCPS specimens, the magnitude of the difference between the two aging evaluations relied on the strategy of IDS employed. Interestingly, the former was caused mainly by a positive effect of aging on the $\mu$ TBS means of silica-coated (SC-PS) specimens from the IDS-F group $(F[1,190.6]=4.886, p=0.020$, $\mathrm{n}^{2}=0.053$ ) (Figure 4), which ultimately led to the highest $\mu$ TBS reported (Table $2 ; 40.6 \pm 14.9 \mathrm{MPa}$ ).
Failure was observed mainly at the adhesivedentin interface for all the evaluated groups. The fewest failures were seen at the adhesive-composite interface and within dentin or composite (Figure 5). The majority of pretesting failures were related to the DDS groups (Table 3).

\section{DISCUSSION}

Glass-ceramic posterior restorations have a good survival rate; however, their prognosis is highly dependent on the strength of the adhesive interface. The weakest link of the interface is the connection of the adhesive to dentin. ${ }^{10,13,15}$ In order to increase the adhesive strength of resin-based materials to dentin in indirect restorations, the concept of IDS was introduced. It was shown in an in vitro study that ceramic laminate veneers could benefit when large surfaces of dentin were exposed. ${ }^{15}$ Freshly exposed dentin is the ideal substrate for dentin bonding. ${ }^{28,29}$ How to apply IDS and how to clean or condition the IDS layer during the luting phase has not been studied to date. ${ }^{32}$

After one week of aging, the magnitude of the difference between the two SC methods depended on
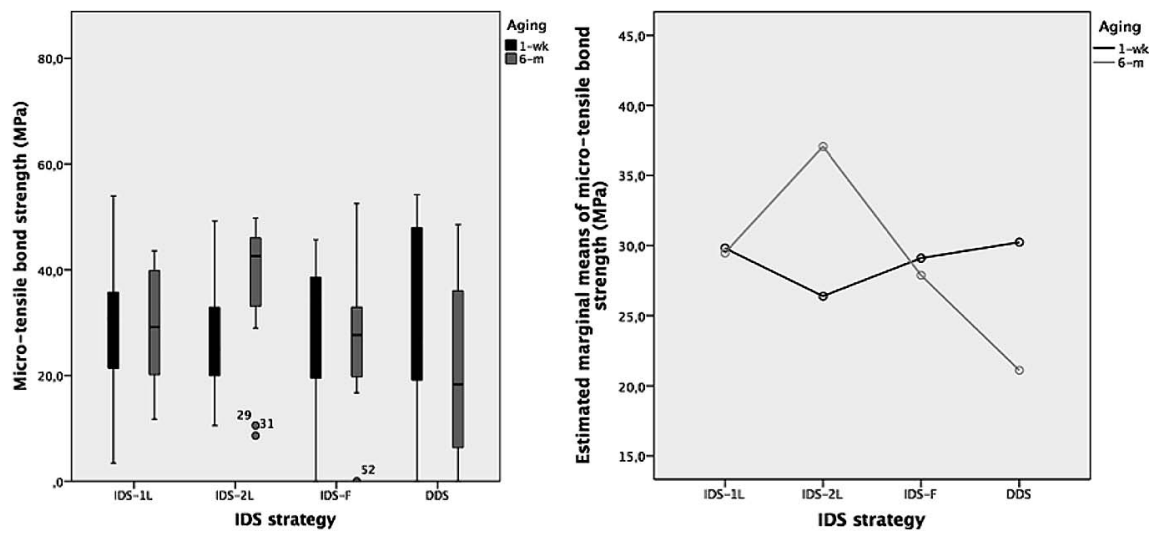

Figure 3. Box plot of microtensile bond strength (MPa) (left) and estimated marginal means of microtensile bond strength (MPa) (right) for the different immediate dentin sealing (IDS) strategies and two evaluation times (1-wk, at one week; 6-m, after six-month aging) when the surface was conditioned with pumice (SC-P). IDS-1L, IDS with one layer of adhesive; IDS-2L, IDS with two layers of adhesive; IDS-F, IDS with one layer of adhesive and one layer of flowable $R B C$; DDS, delayed dentin sealing (control). 

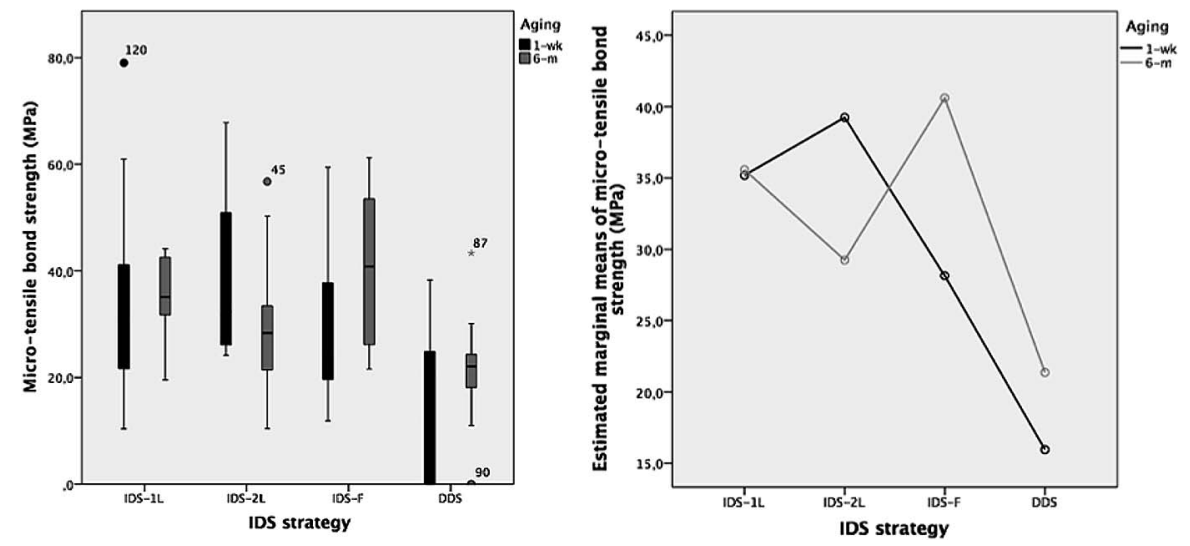

Figure 4. Box plot of microtensile bond strength (MPa) (left) and estimated marginal means of microtensile bond strength (MPa) (right) for the different immediate dentin sealing (IDS) strategies and two evaluation times (1-wk, at one week; 6-m, after six-month aging) when the surface was conditioned with pumice followed by tribochemical silica-coating (SC$P S)$. IDS-1L, IDS with one layer of adhesive; IDS-2L, IDS with two layers of adhesive; IDS-F, IDS with one layer of adhesive and one layer of flowable $R B C$; DDS, delayed dentin sealing (control).

the type of IDS used. This may be explained by the assumption that with only a thin layer of IDS, tribochemical silica coating, which also involves sandblasting, may largely remove the IDS layer, thereby decreasing bond strength. Hence, a thicker IDS layer and silica coating appeared more favorable. This confirms the observations by Stavridakis and others. ${ }^{36}$ They demonstrated the risk of reexposure of dentin after conditioning the preparation, which may be reduced by using a filled bonding agent like Optibond FL (Kerr). In the present study, the three-step etch-and-rinse adhesive Optibond FL was chosen because this adhesive is known for its high filler load and high mechanical strength resulting in higher $\mu$ TBS. ${ }^{10}$ However, after six months, this particular effect was not observed. All different IDS strategies obtained higher bond strengths than the control group (DDS), independent of the SC method used. Therefore, the adhesive application to dentin directly after preparation is important to achieve higher bond strength. Hashimoto and others concluded that bond strength increased with each adhesive coating up to four layers. ${ }^{17}$ Ito and others concluded that simply applying more layers of adhesive could improve the bond strength and the quality of dentin adhesion, especially if the layers were light cured separately. ${ }^{19}$ Multiple layers of adhesive resin are thought to create a thicker adhesive layer without affecting the hybrid-layer quality. The increased bond strength results from an improved stress distribution and increased elasticity of the adhesive layer. ${ }^{4,39}$ However, others claim that each adhesive has its own ideal thickness and that this should be respected in this multilayering technique. ${ }^{7}$ The adhesive system was heated before the application because unpublished data from laboratory studies indicate that it improves $\mu$ TBS.

In contrast to the results of our study, others have observed that bond strength decreased over time, ${ }^{10,16}$ which was attributed to hydrolytic degradation of the adhesive interface. ${ }^{2,25}$ Our results demonstrate that by using IDS, the adhesive interface was stable over time; we could not find a
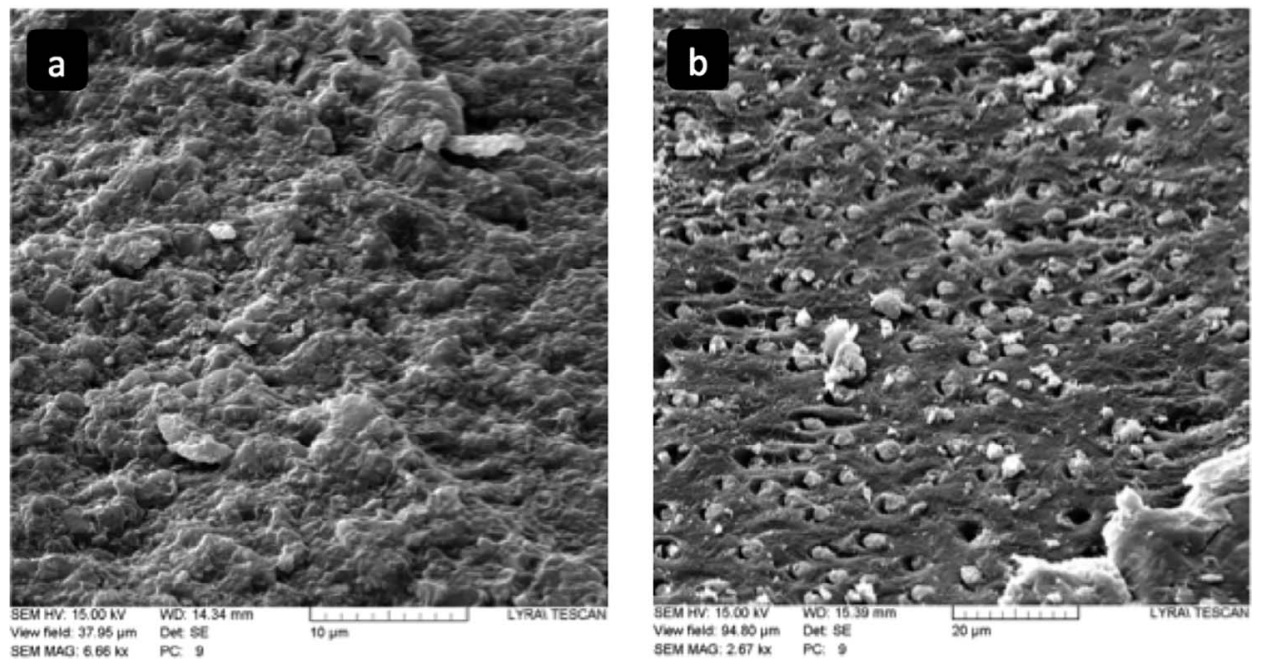

Figure 5. Scanning electron micrographs of fracture surfaces from representative specimens after microtensile bond strength testing. (a): Adhesive failure at the adhesivecomposite interface (IDS-1L, SC-PS, 6-m). (b): Adhesive failure at the adhesive-dentin interface (DDS, SC$P S$, 6-m). Note the exposed dentin tubuli. IDS, immediate dentin sealing; IDS-1L, IDS with one layer of adhesive; IDS-2L, IDS with two layers of adhesive; IDS-F, IDS with one layer of adhesive and one layer of flowable $R B C$; DDS, delayed dentin sealing (control); 1-wk, at one week; 6-m, after six-month aging. 
Table 3: Overview of Pretesting Failures (ptf), Total Number of Specimens (n), and Failure Analysis $(\%)^{a}$

\begin{tabular}{|c|c|c|c|c|c|c|c|}
\hline & ptf & $\mathbf{n}$ & $\mathbf{S}$ & I & B & $B / C$ & C \\
\hline \multicolumn{8}{|l|}{$1 \mathrm{wk}$} \\
\hline IDS-1L + SC-P & 0 & 12 & 11 & 67 & 22 & & \\
\hline IDS-1L + SC-PS & 0 & 12 & & 17 & 17 & & 67 \\
\hline IDS-2L + SC-P & 0 & 12 & & 67 & & & 33 \\
\hline IDS-2L + SC-PS & 0 & 12 & 50 & 33 & & & 17 \\
\hline IDS-F + SC-P & 1 & 12 & & 63 & 25 & 13 & \\
\hline IDS-F + SC-PS & 0 & 12 & 11 & 78 & & & 11 \\
\hline DDS + SC-P & 1 & 12 & & 100 & & & \\
\hline DDS + SC-PS & 4 & 12 & & 100 & & & \\
\hline \multicolumn{8}{|l|}{$6 \mathrm{mo}$} \\
\hline IDS-1L + SC-P & 0 & 12 & 9 & 91 & & & \\
\hline IDS-1L + SC-PS & 0 & 12 & & 80 & & 20 & \\
\hline IDS-2L + SC-P & 0 & 12 & & 76 & & & 25 \\
\hline IDS-2L + SC-PS & 0 & 12 & 25 & 75 & & & \\
\hline IDS-F + SC-P & 1 & 12 & & 33 & 67 & & \\
\hline IDS-F + SC-PS & 0 & 12 & & 67 & & & 33 \\
\hline DDS + SC-P & 3 & 12 & & 80 & 20 & & \\
\hline DDS + SC-PS & 1 & 12 & & 50 & 50 & & \\
\hline \multicolumn{8}{|c|}{$\begin{array}{l}\text { a IDS, immediate dentin sealing; IDS-1L, IDS with one layer of adhesive; } \\
\text { IDS-2L, IDS with two layers of adhesive; IDS-F, IDS with one layer of } \\
\text { adhesive and one layer of flowable RBC; DDS, delayed dentin sealing with } \\
\text { no layer of adhesive (control group); SC, surface conditioning; SC-P, surface } \\
\text { conditioning with pumice rubbing; SC-PC, surface conditioning with pumice } \\
\text { followed by tribochemical silica coating; 1-wk, immediate; } 6-m \text {, aged; ptf, } \\
\text { pretesting failure; } S \text {, failure in dentin; I, failure at the adhesive-dentin } \\
\text { interface; B, failure in adhesive; B/C, failure at the adhesive-composite } \\
\text { interface; } C \text {, failure in composite. }\end{array}$} \\
\hline
\end{tabular}

significantly different effect between the one-week and six-month water storage data. Further investigation is needed to find out if this could be due to an improved resin impregnation associated with an IDS application. According to Magne and others, good bond strength of the definitive restoration to the sealed dentinal surface can be achieved even up to an extended provisionalization phase of 12 weeks. ${ }^{23}$ In this study, the provisionalization phase was three weeks, which, according to Magne and others, could not have affected bond strength. ${ }^{23}$

When surfaces were cleaned using pumice, a stable $\mu$ TBS over time was recorded without a significant difference among the IDS strategies. Surfaces conditioned with tribochemical silica coating demonstrated a more positive effect after six months of aging than the solely pumice-rubbed surfaces. A higher mean bond strength was obtained with a thicker IDS layer compared to a DDS strategy. High mean $\mu$ TBS values and small standard deviations were seen in the IDS-1L group, when specimens were silica coated, after one week
$(35.2 \pm 19.0 \mathrm{MPa})$ and six months $(35.6 \pm 7.3 \mathrm{MPa})$ of aging. Others attributed the improved bond strength recorded using tribochemical silica coating to the additional chemical bonding of the applied silane coupling agent to the silica-coated surface. ${ }^{1,33,37}$

Most failures in the one-week and six-month aged groups were seen at the adhesive-dentin interface; it might be interesting to see whether there would be more differences in bond strength after a longer period of aging. Pretesting failures were seen mainly in the DDS groups, probably due to water uptake of the dentin. In the DDS group, no adhesive interface was created (directly after preparation), while this adhesive interface was necessary to prevent water uptake from the tooth (in the temporary crown phase) ${ }^{18} \mathrm{~A}$ disadvantage of the $\mu$ TBS is not only that the actual "bond" strength measured but also that the strength of the complete assembly, including dentin and composite, ${ }^{9}$ possibly results in a higher strength that surpasses the interfacial strength. Yet $\mu$ TBS is generally accepted as one of the most valid bond strength tests. ${ }^{35}$ According to a review by Qanungo and others ${ }^{32}$ and based on the results of this study, significant differences have been demonstrated between IDS and DDS, this being more in favor of IDS.

\section{CONCLUSIONS}

From this study, the following can be concluded:

1. The $\mu$ TBS recorded for specimens prepared with any type of the three IDS strategies investigated was higher compared to DDS.

2. Cleaning with pumice only or with additional tribochemical silica coating did not affect $\mu$ TBS. When using a silica-coating technique, a thick IDS layer is recommended.

3. A one-layer IDS and surface conditioning involving pumice rubbing with additional tribochemical silica coating appears to be an effective, consistent, durable, and relatively less time consuming IDS procedure.

\section{Acknowledgements}

The authors acknowledge Dr Jan de Munck for his contribution to the study and for helping with the technical aspects of $\boldsymbol{\mu}$ TBS testing. The authors declare that they have no conflict of interest regarding the products investigated in this article. Kerr, Ivoclar Vivadent, and Micerium are gratefully acknowledged for the generous donation of materials for this study.

\section{Regulatory Statement}

This study was conducted in accordance with all the provisions of the local human subjects oversight committee 
guidelines and policies of Rijksuniversity Groningen in the Netherlands.

\section{Conflict of Interest}

The authors did not have any commercial interest in any of the materials used in this study.

(Accepted 7 August 2018)

\section{REFERENCES}

1. Amaral R, Ozcan M, Valandro LF, Balducci I, \& Bottino MA (2008) Effect of conditioning methods on the microtensile bond strength of phosphate monomer-based cement on zirconia ceramic in dry and aged conditions Journal of Biomedical Materials Research. Part B, Applied Biomaterials 85(1) 1-9.

2. Breschi L, Mazzoni A, Ruggeri A, Cadenaro M, Di Lenarda R, \& De Stefano Dorigo E (2008) Dental adhesion review: Aging and stability of the bonded interface Dental Materials 24(1) 90-101.

3. Cardoso MV, de Almeida Neves A, Mine A, Coutinho E, Van Landuyt K, De Munck J, \& Van Meerbeek B (2011) Current aspects on bonding effectiveness and stability in adhesive dentistry Australian Dental Journal 56(Supplement 1) 31-44.

4. Choi KK, Condon JR, \& Ferracane JL (2000) The effects of adhesive thickness on polymerization contraction stress of composite Journal of Dental Research $\mathbf{7 9 ( 3 )}$ 812-817.

5. Dagostin A \& Ferrari M (2002) Effect of resins sealing of dentin on the bond strength of ceramic restorations Dental Materials 18(4) 304-310.

6. Dahl BL (1977) Dentine/pulp reactions to full crown preparation procedures Journal of Oral Rehabilitation 4(3) 247-254.

7. D’Arcangelo C, Vanini L, Prosperi GD, Di Bussolo G, De Angelis F, D'Amario M, \& Caputi S (2009) The influence of adhesive thickness on the microtensile bond strength of three adhesive systems Journal of Adhesive Dentistry 11(2) 109-115.

8. De Goes MF, Giannini M, Di Hipolito V, Carrilho MR, Daronch M, \& Rueggeberg FA (2008) Microtensile bond strength of adhesive systems to dentin with or without application of an intermediate flowable resin layer Brazilian Dental Journal 19(1) 51-56.

9. De Munck J, Luehrs AK, Poitevin A, Van Ende A, \& Van Meerbeek B (2013) Fracture toughness versus microtensile bond strength testing of adhesive-dentin interfaces Dental Materials 29(6) 635-644.

10. De Munck J, Van Landuyt K, Peumans M, Poitevin A, Lambrechts P, Braem M, \& Van Meerbeek B (2005) A critical review of the durability of adhesion to tooth tissue: Methods and results Journal of Dental Research 84(2) $118-132$.

11. Dillenburg AL, Soares CG, Paranhos MP, Spohr AM, Loguercio AD, \& Burnett LH Jr (2009) Microtensile bond strength of prehybridized dentin: Storage time and surface treatment effects Journal of Adhesive Dentistry 11(3) 231-237.
12. Duarte S Jr, de Freitas CR, Saad JR, \& Sadan A (2009) The effect of immediate dentin sealing on the marginal adaptation and bond strengths of total-etch and self-etch adhesives Journal of Prosthetic Dentistry 102(1) 1-9.

13. Dumfahrt H \& Schaffer H (2000) Porcelain laminate veneers: A retrospective evaluation after 1 to 10 years of service: Part II-Clinical results International Journal of Prosthodontics 13(1) 9-18.

14. Falkensammer F, Arnetzl GV, Wildburger A, Krall C, \& Freudenthaler J (2014) Influence of different conditioning methods on immediate and delayed dentin sealing Journal of Prosthetic Dentistry 112(2) 204-210.

15. Gresnigt MM, Cune MS, de Roos JG, \& Ozcan M (2016) Effect of immediate and delayed dentin sealing on the fracture strength, failure type and Weibull characteristics of lithiumdisilicate laminate veneers Dental Materials 32(4) e73-e81.

16. Hashimoto M, Fujita S, Endo K, \& Ohno H (2009) In vitro degradation of resin-dentin bonds with one-bottle selfetching adhesives European Journal of Oral Sciences 117(5) 611-617.

17. Hashimoto M, Sano H, Yoshida E, Hori M, Kaga M, Oguchi H, \& Pashley DH (2004) Effects of multiple adhesive coatings on dentin bonding Operative Dentistry 29(4) 416-423.

18. Hashimoto M, Tay FR, Ito S, Sano H, Kaga M, \& Pashley DH (2005) Permeability of adhesive resin films Journal of Biomedical Materials Research. Part B, Applied Biomaterials 74(2) 699-705.

19. Ito S, Tay FR, Hashimoto M, Yoshiyama M, Saito T, Brackett WW, Waller JL, \& Pashley DH (2005) Effects of multiple coatings of two all-in-one adhesives on dentin bonding Journal of Adhesive Dentistry 7(2) 133-141.

20. Langeland K \& Langeland LK (1970) Pulp reactions to cavity and crown preparation Australian Dental Journal 15(4) 261-276.

21. Magne $P$ (2005) Immediate dentin sealing: A fundamental procedure for indirect bonded restorations Journal of Esthetic and Restorative Dentistry 17(3) 144-154; discussion 155.

22. Magne P, Kim TH, Cascione D, \& Donovan TE (2005) Immediate dentin sealing improves bond strength of indirect restorations Journal of Prosthetic Dentistry 94(6) 511-519.

23. Magne $P$, So WS, \& Cascione D (2007) Immediate dentin sealing supports delayed restoration placement Journal of Prosthetic Dentistry 98(3) 166-174.

24. Morimoto S, Rebello de Sampaio FB, Braga MM, Sesma N, \& Ozcan M (2016) Survival rate of resin and ceramic inlays, onlays, and overlays: A systematic review and meta-analysis Journal of Dental Research 95(9) 985-994.

25. Ozcan M, Barbosa SH, Melo RM, Galhano GA, \& Bottino MA (2007) Effect of surface conditioning methods on the microtensile bond strength of resin composite to composite after aging conditions Dental Materials 23(10) 1276-1282.

26. Pashley DH, Ciucchi B, Sano H, \& Horner JA (1993) Permeability of dentin to adhesive agents Quintessence International 24(9) 618-631. 
27. Pashley EL, Agee KA, Pashley DH, \& Tay FR (2002) Effects of one versus two applications of an unfilled, allin-one adhesive on dentine bonding Journal of Dentistry 30(2-3) 83-90.

28. Pashley EL, Comer RW, Simpson MD, Horner JA, Pashley DH, \& Caughman WF (1992) Dentin permeability: Sealing the dentin in crown preparations Operative Dentistry 17(1) 13-20.

29. Paul SJ \& Scharer P (1997) The dual bonding technique: A modified method to improve adhesive luting procedures International Journal of Periodontics and Restorative Dentistry 17(6) 536-545.

30. Phrukkanon S, Burrow MF, \& Tyas MJ (1999) The effect of dentine location and tubule orientation on the bond strengths between resin and dentine Journal of Dentistry 27(4) 265-274.

31. Poitevin A, De Munck J, Van Landuyt K, Coutinho E, Peumans M, Lambrechts P, \& Van Meerbeek B (2007) Influence of three specimen fixation modes on the microtensile bond strength of adhesives to dentin Dental Materials Journal 26(5) 694-699.

32. Qanungo A, Aras MA, Chitre V, Mysore A, Amin B, \& Daswani SR (2016) Immediate dentin sealing for indirect bonded restorations Journal of Prosthodontic Research 60(4) $240-249$.

33. Radovic I, Monticelli F, Goracci C, Cury AH, Coniglio I, Vulicevic ZR, Garcia-Godoy F, \& Ferrari M (2007) The effect of sandblasting on adhesion of a dual-cured resin composite to methacrylic fiber posts: Microtensile bond strength and SEM evaluation Journal of Dentistry 35(6) 496-502.
34. Rocca GT, Gregor L, Sandoval MJ, Krejci I, \& Dietschi D (2012) In vitro evaluation of marginal and internal adaptation after occlusal stressing of indirect class II composite restorations with different resinous bases and interface treatments: Post-fatigue adaptation of indirect composite restorations Clinical Oral Investigations 16(5) 1385-1393.

35. Sirisha K, Rambabu T, Ravishankar Y, \& Ravikumar P (2014) Validity of bond strength tests: A critical reviewPart II Journal of Conservative Dentistry 17(5) 420-426.

36. Stavridakis MM, Krejci I, \& Magne P (2005) Immediate dentin sealing of onlay preparations: Thickness of precured dentin bonding agent and effect of surface cleaning Operative Dentistry 30(6) 747-757.

37. Sun R, Suansuwan N, Kilpatrick N, \& Swain M (2000) Characterisation of tribochemically assisted bonding of composite resin to porcelain and metal Journal of Dentistry 28(6) 441-445.

38. van den Breemer CR, Gresnigt MM, \& Cune MS (2015) Cementation of glass-ceramic posterior restorations: A systematic review BioMed Research International 2015 148954.

39. Van Meerbeek B, Willems G, Celis JP, Roos JR, Braem M, Lambrechts P, \& Vanherle G (1993) Assessment by nanoindentation of the hardness and elasticity of the resindentin bonding area Journal of Dental Research 72(10) 1434-1442.

40. Yoshiyama M, Matsuo T, Ebisu S, \& Pashley D (1998) Regional bond strengths of self-etching/self-priming adhesive systems Journal of Dentistry 26(7) 609-616. 